\title{
Efficient approximations of neutrino physics for three-dimensional simulations of stellar core collapse
}

\author{
Matthias Liebendörfer* \\ University of Basel \\ E-mail: matthias.liebendoerfer@unibas.ch

\section{Ue-Li Pen, Christopher Thompson} \\ CITA, University of Toronto \\ E-mail: penecita.utoronto.ca, thompsonecita.utoronto.ca
}

\begin{abstract}
Neutrino transport in spherically symmetric models of stellar core collapse and bounce has achieved a technically complete level, rewarded by the agreement among independent groups that a multi-dimensional treatment of the fluid-instabilities in the post-bounce phase is indispensable to model supernova explosions. While much effort is required to develop a reliable neutrino transport technique in axisymmetry, we explore neutrino physics approximations and parameterizations for an efficient three-dimensional simulation of the fluid-instabilities in the shock-heated matter that accumulates between the accretion shock and the protoneutron star. We demonstrate the reliability of a simple parameterization scheme in the collapse phase and extend our 3D magneto-hydrodynamical collapse simulations to a preliminary postbounce evolution. The growth of magnetic fields is investigated.
\end{abstract}

International Symposium on Nuclear Astrophysics - Nuclei in the Cosmos - IX

25-30 June 2006

CERN

\footnotetext{
${ }^{*}$ Speaker.
} 


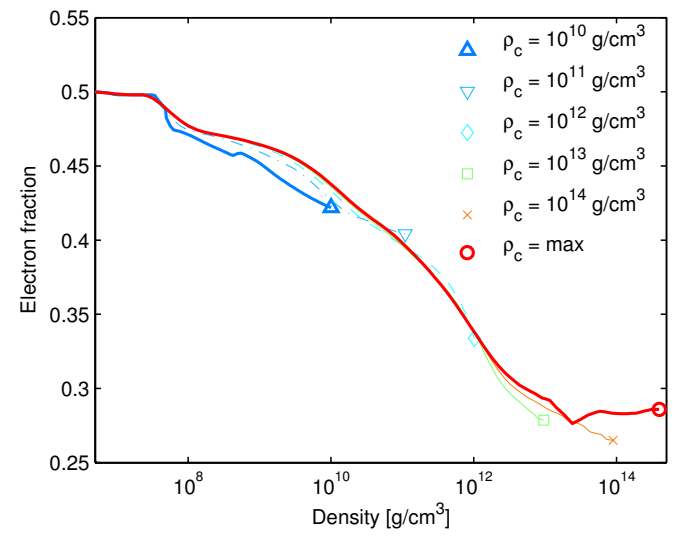

Figure 1: Electron fraction profiles during core collapse in model G15 [1]. Each line shows $Y_{e}$ as a function of density at a given time. As $Y_{e}(\rho)$ is only a weak function of time and because the accuracy is most important at core-bounce, the parameterization of $Y_{e}$ is conveniently based on the bounce profile labelled by a circle.

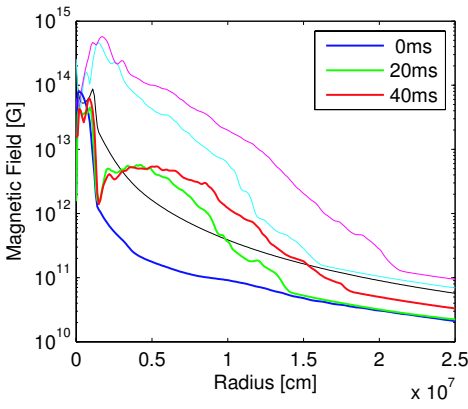

Figure 2: Strength of the magnetic field at 0 $\mathrm{ms}, 20 \mathrm{~ms}$, and $40 \mathrm{~ms}$ after bounce in two different models. Model 1 (black, cyan, magenta), which starts with a poloidal field and $\Omega=2 \pi$ $\mathrm{rad} / \mathrm{s}$, shows rapid field amplification by winding at the surface of the protoneutron star. This effect is negligible in model 2 (blue, green, red), which started with a toroidal field and $\Omega=0.05$ $\mathrm{rad} / \mathrm{s}$.

\section{Parameterization of neutrino physics and comparison with reference data}

In multi-dimensional simulations of stellar core collapse, rotating progenitors, general relativity, and magnetic fields have been investigated. Only few recent multi-dimensional collapse simulations made the effort to include neutrino physics. These schemes are either very computationally expensive $[2,3]$ or rely on simplifications of the neutrino transport and its microphysics $[4,5]$. The dynamics of core collapse is dominated by electron pressure. Dynamical simulations are only realistic if they take electron captures into account. At increasing densities, the electron captures get inhibited by neutrino phase space blocking so that the ability to thermalize and emit the produced neutrinos significantly contributes to the determination of the final electron fraction $\left(Y_{e}\right)$ in the inner core. The lower the $Y_{e}$, the smaller is the mass of the core that bounces when nuclear densities are reached and the smaller is also the initial energy imparted to the outgoing shock. These well-known relationships ask for a careful inclusion of neutrino physics in simulations of stellar core collapse. The direct solution of the Boltzmann neutrino transport equation, however, has only been achieved under the assumption of spherical symmetry (Ref. [6] and references therein).

Three-dimensional models must rely on approximations of the neutrino transport to be treatable by current computers. A simple and computationally efficient parameterization of the deleptonization in the collapse phase is based on a tabulation of $Y_{e}$ as a function of density. A spherically symmetric model with Boltzmann neutrino transport [1] shows that this function is fairly independent of time so that it can be used throughout the collapse phase (see Fig. 1). Once the deleptonization is known, an estimate of entropy changes and neutrino stress can be deduced [7]. The comparison of the full-domain 3D parameterized runs with the spherically symmetric general relativistic reference model G15 shows that the 1D results of core collapse are accurately reproduced by a 3D run if it is launched from a non-rotational progenitor (see Fig. 3). The parameterized 

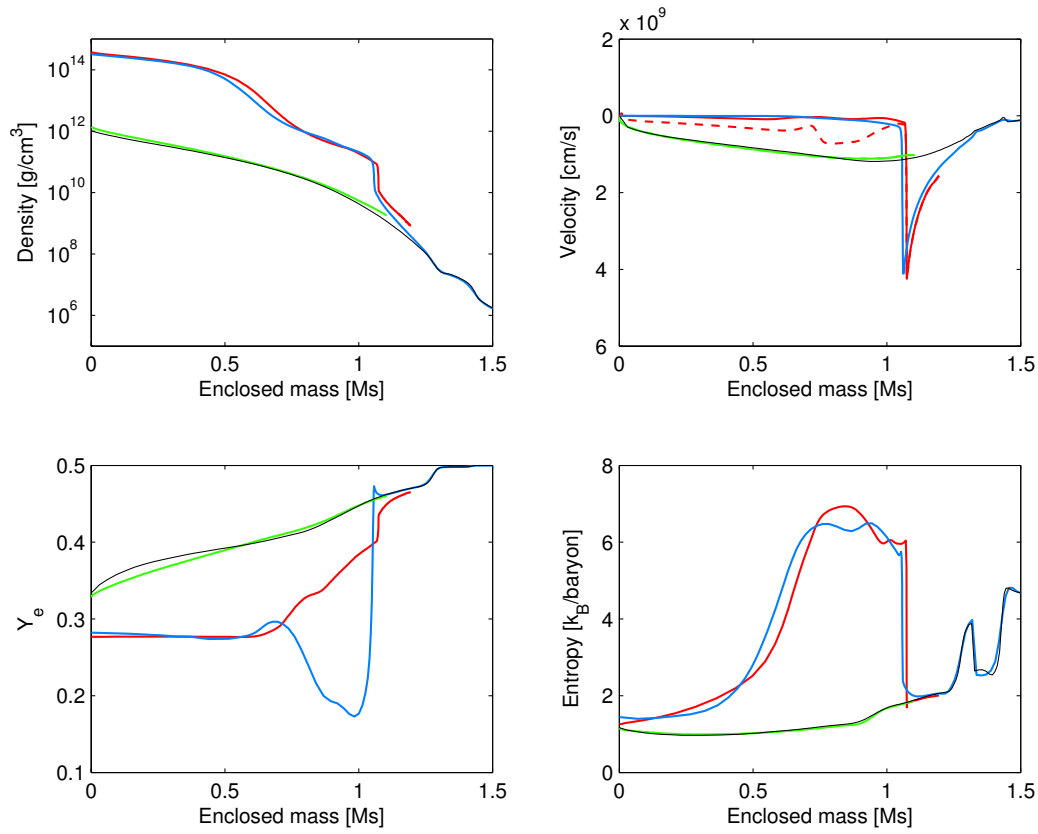

Figure 3: Comparison of the outcome of a parameterized 3D simulation with model G15, which is based on general relativistic three-flavour Boltzmann neutrino transport [1]. From the upper left to the lower right we compare as a function of enclosed mass: the density-, the velocity-, the $Y_{e^{-}}$, and the entropy profiles. The green and red lines represent the spherically averaged profiles from the 3D run at $5 \mathrm{~ms}$ before and $5 \mathrm{~ms}$ after bounce, respectively. The black and blue lines represent the G15 reference data at corresponding times. Excellent agreement is found in all four quantities-with one exception: This simple parameterized neutrino leakage cannot model the neutrino burst. The neutrino burst causes a prominent $Y_{e}$-dip and additional cooling in the G15 data.

neutrino physics presents a significant improvement with respect to adiabatic simulations and may even rival with neutrino transport schemes that neglect neutrino-electron scattering. However, the accuracy breaks down with the launch of the neutrino burst at a few milliseconds after bounce. With the currently implemented scheme, accretion flows in the postbounce phase deleptonize only down to $Y_{e} \sim 0.3$ instead of $Y_{e} \sim 0.15$. Neutrino heating is neglected altogether. Better neutrino physics approximations are required after core collapse.

\section{Three-dimensional simulations with magnetic fields}

The 3D simulations are based on a simple and fast cosmological MHD code [8] which has been parallelized, improved and adapted to the supernova context. A realistic equation of state [9] is used and gravity is implemented by a spherically symmetric mass integration which includes general relativistic corrections [10]. The simulations are launched from a $15 \mathrm{M}_{\odot}$ progenitor model [11]. The 3D computational domain consists of a central region of $600 \mathrm{~km}^{3}$, treated in equidistant Cartesian coordinates with a resolution of $1 \mathrm{~km}$. Outside of the neutron star, this resolution is comparable to or better than the resolution chosen in 1D simulations (cf. the shock widths in Fig. 3). The 3D computational domain is embedded in a larger spherically symmetrical computational domain treated by a $1 \mathrm{D}$ hydrodynamics code. In the pre-collapse configuration, an initial 


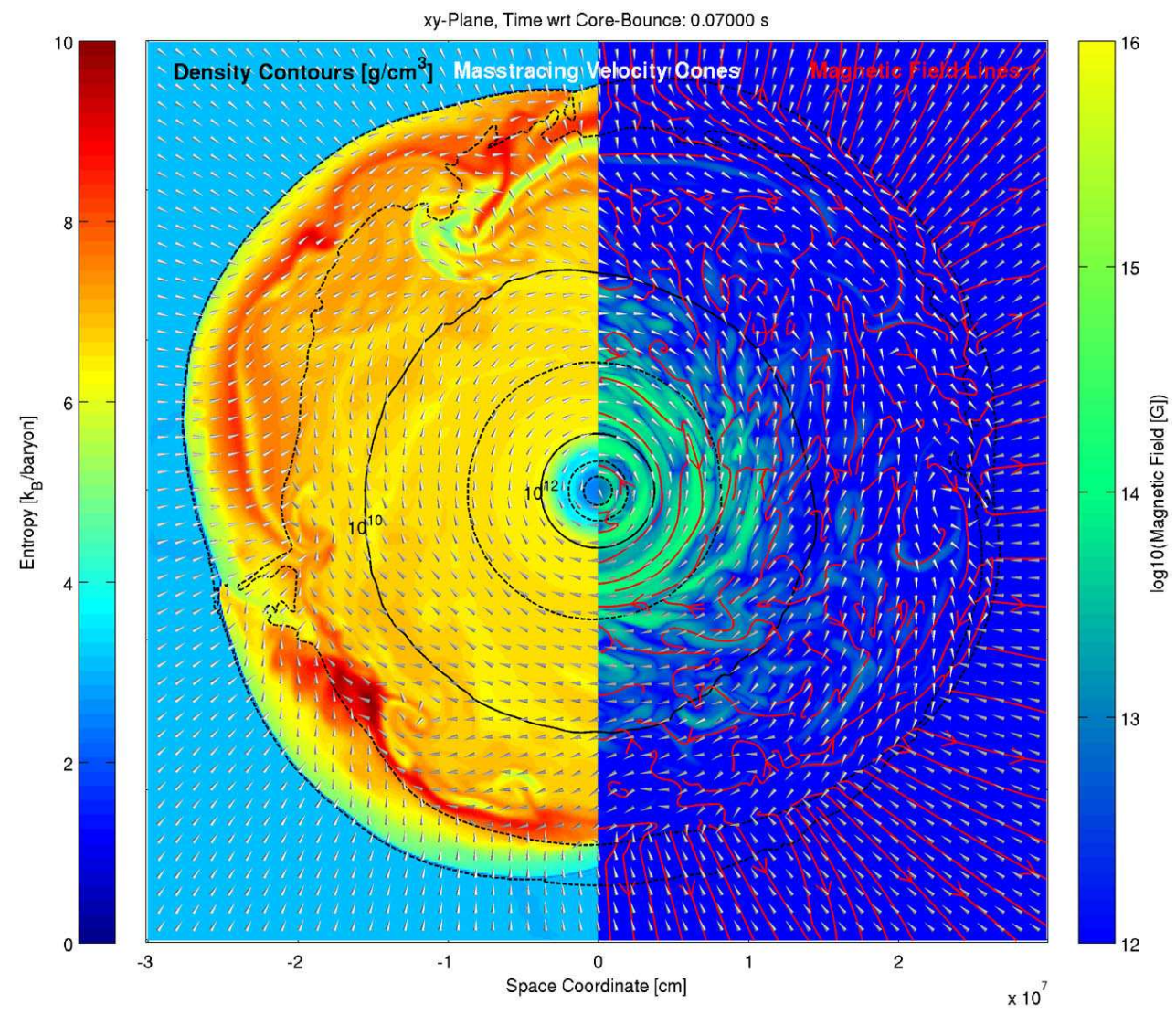

Figure 4: Slice through the equatorial plane of a 3D simulation at $70 \mathrm{~ms}$ after bounce. On the left hand side the background color indicates the entropy per baryon, and on the right hand side the magnetic field strength. Density contours are drawn in black, and magnetic field lines in red. The white cones align with the $3 \mathrm{D}$ velocity field. In this rotating model, instabilities of the standing accretion front develop more strongly in the equatorial plane than in the polar direction.

angular momentum is assigned to the spherical mass shells according to an angular velocity of $2 \pi$ $\mathrm{rad} / \mathrm{s}$ and a shellular quadratic cutoff at $500 \mathrm{~km}$ radius. The angular momentum is assumed to be conserved until the infalling layers enter the 3D computational domain. The initial poloidal magnetic field is derived from a vector potential whose norm scales with the square root of the density. It is set to produce a field strength of $5 \times 10^{9}$ Gauss at a reference density of $5 \times 10^{7} \mathrm{~g} / \mathrm{cm}^{3}$.

As the duration of the collapse phase is no longer than a fraction of a rotational period, the magnetic field does not significantly wind up during collapse. Its amplification by about two orders of magnitude is due to the compression of field lines in the condensing matter. A poloidal initial field develops a biconical shape, focussed on the protoneutron star at the origin. Toroidal initial fields tend to remain toroidal throughout collapse. Figure 2 compares a fast rotating model with poloidal field to a model where initial rotation and magnetic fields are set to the values suggested in Ref. [12]. A different amplification of the field strength by winding becomes evident close to the surface of the protoneutron star. The offset between the field strengths of simultaneous profiles at larger distances, however, stem from the initial configuration ${ }^{1}$. After bounce, the declining

\footnotetext{
${ }^{1}$ The vector potentials used to generate the initial fields align differently with the gradient of the density to which
} 
strength of the bounce-shock results in a negative entropy gradient. The hot matter layered around the protoneutron star becomes convectively unstable on a short time scale. This causes the magnetic field lines in this region to entangle (see Fig. 4) and to grow further.

\section{Acknowledgments}

This work is funded by the Swiss National Science Foundation, grant No. PP002-106627. The simulations have been carried out on the Athena cluster at the University of Basel and on the McKenzie cluster [13] which was funded by the Canada Foundation for Innovation and the Ontario Innovation Trust.

\section{References}

[1] M. Liebendörfer, M. Rampp, H.-T. Janka, and A. Mezzacappa, Supernova Simulations with Boltzmann Neutrino Transport: A Comparison of Methods, ApJ 620 (2005) 840-860.

[2] R. Buras, M. Rampp, H.-T. Janka, and K. Kifonidis, Improved models of stellar core collapse and still no explosions: What is missing?, Phys. Rev. Lett. 90 (2003), no. 24241101.

[3] L. Dessart, A. Burrows, C. D. Ott, E. Livne, S.-Y. Yoon, and N. Langer, Multidimensional Simulations of the Accretion-induced Collapse of White Dwarfs to Neutron Stars, ApJ 644 (2006) 1063-1084.

[4] K. Kotake, S. Yamada, and K. Sato, Gravitational radiation from axisymmetric rotational core collapse, Phys. Rev. D 68 (2003) 044023-+.

[5] C. L. Fryer and M. S. Warren, The Collapse of Rotating Massive Stars in Three Dimensions, ApJ 601 (2004) 391-404.

[6] A. Mezzacappa, The Core Collapse Supernova Mechanism: Current Models, Gaps, and the Road Ahead, in ASP Conf. Ser. 342: 1604-2004: Supernovae as Cosmological Lighthouses (M. Turatto, S. Benetti, L. Zampieri, and W. Shea, eds.), pp. 175-+, 2005.

[7] M. Liebendörfer, A Simple Parameterization of the Consequences of Deleptonization for Simulations of Stellar Core Collapse, ApJ 633 (2005) 1042-1051.

[8] U.-L. Pen, P. Arras, and S. Wong, A Free, Fast, Simple, and Efficient Total Variation Diminishing Magnetohydrodynamic Code, ApJS 149 (2003) 447-455.

[9] J. M. Lattimer and F. D. Swesty, A generalized equation of state for hot, dense matter, Nucl. Phys. A 535 (1991) 331-376.

[10] A. Marek, H. Dimmelmeier, H.-T. Janka, E. Müller, and R. Buras, Exploring the relativistic regime with Newtonian hydrodynamics: an improved effective gravitational potential for supernova simulations, A\&A 445 (2006) 273-289.

[11] S. E. Woosley and T. A. Weaver, The evolution and explosion of massive stars. ii. explosion hydrodynamics and nucleosynthesis, ApJS 101 (1995) 181.

[12] A. Heger, S. E. Woosley, and H. C. Spruit, Presupernova Evolution of Differentially Rotating Massive Stars Including Magnetic Fields, ApJ 626 (2005) 350-363.

[13] J. Dubinski, R. J. Humble, C. Loken, U.-L. Pen, and P. G. Martin, Mckenzie: A teraflops linux beowulf cluster for computational astrophysics, in Proc. of the 17th Annual International Symposium on High Performance Computing Systems and Applications, May 11-14, Sherbrooke, PQ, 2003.

they were scaled. Thus, the toroidal case starts with a smaller field strength in the outer layers than the poloidal case. 